
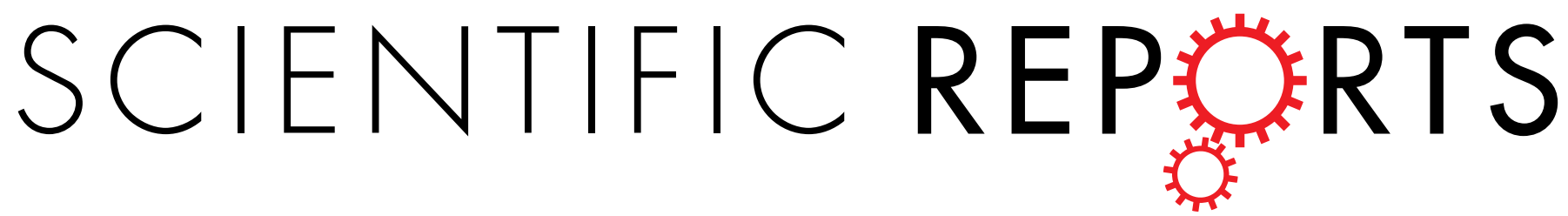

OPEN

Received: 19 February 2016

Accepted: 17 May 2016

Published: 03 June 2016

\title{
Riems influenza a typing array (RITA): An RT-qPCR-based low density array for subtyping avian
} and mammalian influenza a viruses

\author{
Bernd Hoffmann, Donata Hoffmann, Dinah Henritzi, Martin Beer \& Timm C. Harder
}

Rapid and sensitive diagnostic approaches are of the utmost importance for the detection of humans and animals infected by specific influenza virus subtype(s). Cascade-like diagnostics starting with the use of pan-influenza assays and subsequent subtyping devices are normally used. Here, we demonstrated a novel low density array combining 32 TaqMan ${ }^{\circledR}$ real-time RT-PCR systems in parallel for the specific detection of the haemagglutinin (HA) and neuraminidase (NA) subtypes of avian and porcine hosts. The sensitivity of the newly developed system was compared with that of the pan-influenza assay, and the specificity of all RT-qPCRs was examined using a broad panel of 404 different influenza $A$ virus isolates representing 45 different subtypes. Furthermore, we analysed the performance of the RT-qPCR assays with diagnostic samples obtained from wild birds and swine. Due to the open format of the array, adaptations to detect newly emerging influenza A virus strains can easily be integrated. The RITA array represents a competitive, fast and sensitive subtyping tool that requires neither new machinery nor additional training of staff in a lab where RT-qPCR is already established.

Influenza A viruses (IAVs) cause infections in various avian and several mammalian species, including humans ${ }^{1}$. The genomes of IAVs are composed of eight negative sensed single stranded RNA segments ${ }^{2}$. Due to the segmented nature of the genome combinatorial exchanges of genome segments, so-called reassortments may occur when different IAVs replicate in the same host cell. This phenomenon significantly increases IAV genotypic variability and may also contribute to ad hoc shifts in viral phenotypes. Two glycoprotein species located in the viral membranes of IAVs, the haemagglutinin (HAs) and the neuraminidases (NAs), represent the major antigenic determinants that define the subtype of the virus ${ }^{2}$. Reassortment events that changed a combination of HA and NA glycoproteins and created a novel antigenic phenotype in an immunologically naïve human population have been the basis of pandemic, i.e., global waves of human IAV infections ${ }^{3}$. Only a subset of all known IAV subtypes infect mammalian species, whereas avian metapopulations perpetuate the full spectrum of 16 different HA and 9 NA entities ${ }^{4-6}$. The only exceptions are the recently described bat influenza viruses H17N10 and H18N11 that were up to now detected in bats only ${ }^{7,8}$.

The symptoms, if any, of IAV induced human disease vary from mild to severe respiratory disorders. However, rarely occurring severe systemic disorders with substantial fatality rates have been reported, as well ${ }^{9}$. In temperate climates, there is a sharply defined human influenza season, during which a total of 1 billion people are affected annually ${ }^{10}$. Influenza A-related diseases induced in susceptible livestock, particularly poultry and swine, are highly variable and may have economic implications due to animal losses, reduced production rates and/or trade restrictions. The severity of influenza infections in animals is, to some extent, related to the species, age and constitution of the host, but there are also influential viral determinants of pathogenicity. This is particularly true for highly pathogenic forms of avian influenza (AI) caused by certain lineages of the AIV subtypes H5 and H7. Avian infections with either of these subtypes, independent of their pathogenicity, are notifiable and call upon rigorous restriction and eradication measures (e.g. "stamping out") ${ }^{11}$.

Due to the potentially far-reaching consequences of the detection of certain IAV subtypes, a rapid and reliable diagnostic subtyping assay is of paramount importance. Virus subtyping directly from original sample material would considerably reduce the time until diagnosis. Here, we describe a very broad molecular subtyping assay 


\begin{tabular}{|l|c|c|c|c|c|}
\hline & human & avian & Porcine & other mammals & Total \\
\hline H1 & 21 & 10 & 23 & - & 54 \\
\hline H2 & 1 & 17 & - & - & 18 \\
\hline H3 & 8 & 24 & 7 & 1 & 40 \\
\hline H4 & - & 17 & - & - & 17 \\
\hline H5 & 3 & 83 & - & 2 & 88 \\
\hline H6 & - & 61 & - & - & 61 \\
\hline H7 & - & 34 & - & 1 & 35 \\
\hline H8 & - & 3 & - & - & 3 \\
\hline H9 & - & 31 & - & - & 31 \\
\hline H10 & - & 29 & - & - & 29 \\
\hline H11 & - & 11 & - & - & 11 \\
\hline H12 & - & 1 & - & - & 1 \\
\hline H13 & - & 9 & - & 1 & 10 \\
\hline H14 & - & 1 & - & - & 1 \\
\hline H15 & - & 1 & - & - & 1 \\
\hline H16 & - & 4 & - & - & 4 \\
\hline Total & 33 & 336 & 30 & 5 & 404 \\
\hline
\end{tabular}

Table 1. Condensed overview of the haemagglutinin subtype of viral isolates used to validate RITA.

\begin{tabular}{|l|c|c|c|c|c|c|c|c|c|c|}
\hline & N1 & N2 & N3 & N4 & N5 & N6 & N7 & N8 & N9 & total \\
\hline H1 & 47 & 7 & - & - & - & - & - & - & - & 54 \\
\hline H2 & - & 2 & 10 & - & - & - & - & - & 6 & 18 \\
\hline H3 & 1 & 22 & - & - & - & 3 & - & 14 & - & 40 \\
\hline H4 & - & 1 & - & - & - & 16 & - & - & - & 17 \\
\hline H5 & 60 & 9 & 15 & - & - & 1 & - & - & 3 & 88 \\
\hline H6 & 7 & 41 & - & 1 & 3 & - & - & 8 & 1 & 61 \\
\hline H7 & 8 & 2 & 7 & 2 & - & - & 16 & - & - & 35 \\
\hline H8 & - & - & 1 & 2 & - & - & - & - & - & 3 \\
\hline H9 & - & 31 & - & - & - & - & - & - & - & 31 \\
\hline H10 & - & 1 & - & 6 & - & - & 21 & 1 & - & 29 \\
\hline H11 & 3 & 1 & - & - & - & 2 & - & - & 5 & 11 \\
\hline H12 & - & - & - & - & 1 & - & - & - & - & 1 \\
\hline H13 & - & 4 & - & - & - & 4 & - & 2 & - & 10 \\
\hline H14 & - & - & - & - & 1 & - & - & - & - & 1 \\
\hline H15 & - & - & - & - & - & - & - & - & 1 & 1 \\
\hline H16 & - & - & 4 & - & - & - & - & - & - & 4 \\
\hline Total & 126 & 121 & 37 & 11 & 5 & 26 & 37 & 25 & 16 & 404 \\
\hline
\end{tabular}

Table 2. Summary of the neuraminidase subtypes of the viral isolates used to validate RITA.

system based on reverse transcription quantitative PCR (RT-qPCR) in a multiwell layout that is easily prepared and runs on standard lab equipment. By using a hydrolysis probe technique (TaqMan ${ }^{\circledR}$ ), one of the most powerful and widespread methodologies in diagnostic microbiology ${ }^{12}$ was applied. The format is referred to as the Riems Influenza A Typing Array, abbreviated RITA. The RITA assay is the first system that combines simple duplex $\mathrm{TaqMan}^{\circledR}$ reactions into one diagnostic tool, without the need for further equipment for detection or identification of $14 \mathrm{HA}$ and 9 NA subtypes of influenza A viruses. More than 400 IAV isolates and clinical material from 63 swab samples were successfully subtyped to qualify this protocol as eminently suitable for routine procedures.

\section{Materials and Methods}

Viruses. Samples from 404 influenza viral strains representing 16 HA and 9 NA subtypes pre-typed (either serologically or by sequencing) at the National Reference Laboratory for Avian Influenza (NRL-AI, FLI) were used for analytical validation of the newly developed assays. These isolates are referred to as the "validation panel" throughout this manuscript. Tables 1 and 2 provide a condensed overview of the subtypes and species of origin, and supplemental Table S1 (available online) details the individual viral strains. Furthermore, to assess the applicability of RITA to investigate field samples containing various loads of IAV RNA, 62 swabs from wild birds (originating from wild bird monitoring) and swine (diagnostic samples from diseased swine herds) were tested. Sanger 
sequencing of amplicons generated from the field sample RNAs by pan-HA and pan NA RT-PCR-protocols ${ }^{13,14}$ (Henritzi et al. under review) was used to verify the RITA subtyping results.

Primers and probes. The pan-influenza A IAV-M1.2 assay ${ }^{15}$ was implemented in RITA as a control for the presence of IAV RNA in the sample tested and to provide quantification cycle values $(\mathrm{Cq})$ for comparison to other HA- and NA-specific RT-qPCRs in the array. By in silico analyses of publicly available IAV sequences, primers and probes specific for the different HA and NA subtypes were identified. The primer and probe sets used in this study are detailed in Table 3. Oligos were synthesized by Metabion GmbH (Martinsried, Germany) and stored at $-20^{\circ} \mathrm{C}$ until use.

RNA extraction. Viral RNA was extracted from supernatants of infected MDCK cell cultures or allantoic fluids of embryonated chicken eggs using the MagNA Pure LC Total Nucleic Acid Isolation Kit (Roche, Penzberg, Germany), according to the manufacturer's instructions, on a MagNA Pure LC 2.0 system (Roche). Clinical material (swab samples) was extracted manually using the Qiagen Viral RNA kit (Qiagen, Hilden, Germany) or by the Qiagen MagAttract Kit operated on a KingFisher Biosprint96 device (Qiagen).

Individual RT-qPCRs and plate arrangement. All individual RT-qPCRs were performed on the Bio-Rad CFX 96 real-time PCR (Bio-Rad, Munich, Germany) platform in a 96-well format using the AgPath-ID One-Step kit (Applied Biosystems, Foster City, CA). Using a heterologous internal control system ${ }^{16}$, the integrity of the reagents was assured, and inhibition phenomena were excluded. The composition of a single reaction of $12.5 \mu \mathrm{l}$ was as follows: $1 \mu \mathrm{l}$ of RNase-free water, $6.25 \mu \mathrm{l}$ of $2 \mathrm{x}$ RT-PCR buffer, $0.5 \mu \mathrm{l}$ of RT-PCR Enzyme Mix, $0.25 \mu l$ of internal control template, $1 \mu \mathrm{l}$ of primer-probe-mix for the internal control and $1 \mu \mathrm{l}$ of IAV specific primer-probe-mix. Finally, $2.5 \mu$ l of RNA template was added. In its current lay-out, 32 wells (i.e., four eight-well stripes) of a 96 well PCR plate are used per RITA analysis. Thus, running three samples in parallel on one 96-well plate is possible. A typical lay-out of the 32 wells is depicted in supplemental Fig. S1, available online. During the setup of a plate, RNase-free water $(0.5 \mu \mathrm{l})$, the IAV specific primer-probe-mix $(1 \mu \mathrm{l})$ and the primer-probe-mix for the internal control $(1 \mu \mathrm{l})$ were added to each individual well. At this stage of preparation, storage of the plate at $-20^{\circ} \mathrm{C}$ is possible, and bulk production of plates is advisable. After thawing the required amount of plates, the master mixes for each sample were prepared, which were composed of the RT-PCR buffer, the enzyme mix, the internal control RNA, residual water and the extracted viral RNA. Ten microliters of this mixture was added to each of the 32 wells of the test using a multi-channel pipette. Thus, a total of $80 \mu \mathrm{l}$ of extracted RNA is required per sample to feed the 32 wells for a complete characterization. For PCR amplification, the following temperature profile was applied: $10 \mathrm{~min}$ at $45^{\circ} \mathrm{C}$ (reverse transcription) and $10 \mathrm{~min}$ at $95^{\circ} \mathrm{C}$ (inactivation of the reverse transcriptase/activation Taq polymerase), followed by 45 cycles of $15 \mathrm{sec}$ at $95^{\circ} \mathrm{C}$ (denaturation), $20 \mathrm{sec}$ at $56^{\circ} \mathrm{C}$ (annealing) and $30 \mathrm{sec}$ at $72^{\circ} \mathrm{C}$ (elongation). Fluorescence values (FAM, HEX) were collected during the annealing step.

In order to confirm the integrity of all of the target- and internal control-specific primer-probe-mixtures a positive control containing a mixture of RNA of all $\mathrm{H}$ and $\mathrm{N}$ subtypes targeted (panIAV-PC) is recommended. The panIAV-PC is a mixture of IAV-RNA from all IAV subtypes analysed in the array. However, based on the internal control system and the inclusion of the pan-influenza A IAV-M1.2 assay ${ }^{15}$, the application of RITA without the co-analysis of panIAV-PC is also valid. The analysis of RNase free water as a no template control (NTC) may identify cross-contamination of the master mix. Analysis of the NTC and the panIAV-PC was required after a new batch of arrays was produced, or the arrays were stored in the freezer for a prolonged period of time (more than 4 weeks) before use.

\section{Results}

RT-qPCRs and construction of a 32-well PCR array. By in silico analysis of published sequence data, primer and probes for the generic detection of HA subtypes H1 to H13 and H16 and all 9 NA subtypes were selected with the aim of detecting the broadest possible spectrum within a given subtype. To this end, various RT-qPCRs were newly developed, and their performance, analysed. In addition, published RT-qPCR subtyping protocols were evaluated, as well. Finally, the best performing assays were combined into the low density array

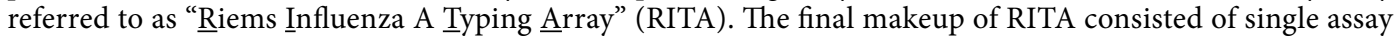
detection of subtypes $\mathrm{H} 4, \mathrm{H} 6, \mathrm{H} 8, \mathrm{H} 9, \mathrm{H} 11, \mathrm{H} 12, \mathrm{H} 13$ and $\mathrm{H} 16$; two assays for $\mathrm{H} 1, \mathrm{H} 2, \mathrm{H} 3, \mathrm{H} 5$ and $\mathrm{H} 10$ detection; and three assays for $\mathrm{H} 7$ detection. NA subtyping was performed by single assays, except for the N3 subtype, which required two assays. This process resulted in the ultimate 32 well format RITA, which was evaluated using a selected set of 404 IAV isolates (detailed in supplemental Table S1). All samples were also run in the pan-influenza IAV-M1.2-assay ${ }^{15}$ included in the 32 well RITA to verify the presence of IAV RNA in general and to obtain an estimation of the viral genome load of each individual sample by generic amplification of a highly conserved region of genome segment 7 of all IAV isolates ${ }^{15}$.

Analytical sensitivity of RT-qPCRs. The analytical sensitivity of each single assay of RITA was evaluated using a dilution series of matching viral RNAs and compared with the Cq-values of the respective RNA obtained by the generic-influenza IAV-M1.2 test. The analyses showed that all subtype specific assays were similar sensitive as the generic-influenza assay. At the maximum 10-fold less viral RNA was detected, using the serotype specific assays compared to the generic pan-influenza test. In some cases, a better analytical sensitivity for the serotype specific assay was measured (data available upon request).

Analytical specificity of RITA. Analytical specificity was assessed by the determination of inclusivity and exclusivity on a validation panel of 404 different IAV isolates. The results are summarized in Table 4 . As an example, "H1-subtype mix 3" detected 51 out of $54 \mathrm{H} 1$ virus isolates correctly, whereas "H1 mix 27" detected 53 out of 54 H1 RNAs. Isolates that were not detected differed between the two assays: "H1 mix 3" did not detect 


\begin{tabular}{|c|c|c|}
\hline Designation & Sequence $5^{\prime} \Rightarrow 3^{\prime}$ & $\begin{array}{l}\text { Concentration of primer and } \\
\text { probes in the primer-probe-mix }\end{array}$ \\
\hline \multicolumn{3}{|l|}{ Pan-IAV assay } \\
\hline IAV-M1.2 & Hoffmann et al. ${ }^{15}$ & \\
\hline IAV-M1-F & AGA TGA GTC TTC TAA CCG AGG TCG & $20 \mu \mathrm{M}$ \\
\hline IAV-M1.1-R & TGC AAA AAC ATC TTC AAG TYT CTG & $15 \mu \mathrm{M}$ \\
\hline IAV-M1.2-R & TGC AAA GAC ACT TTC CAG TCT CTG & $15 \mu \mathrm{M}$ \\
\hline IAV-M1-FAM & FAM-TCA GGC CCC CTC AAA GCC GA-BHQ1 & $2.5 \mu \mathrm{M}$ \\
\hline \multicolumn{3}{|l|}{ H1 assays } \\
\hline IAV-H1-Mix 3-FAM & (this study) & \\
\hline IAV-H1-115F & ACA CAA TAT GTA TAG GYT AHC ATG C & $20 \mu \mathrm{M}$ \\
\hline IAV-H1-199R & GAG TGT GTY ACT GTY ACA TTC TT & $20 \mu \mathrm{M}$ \\
\hline IAV-H1-147FAM & FAM-TCD ACM GAC ACT GTW GAC ACA GTA CTN GA-BHQ1 & $5 \mu \mathrm{M}$ \\
\hline IAV-H1-Mix 27-FAM & (this study) & \\
\hline IAV-H1-1078F & AGG AAT GTC CCR TCY ATT CAA TC & $20 \mu \mathrm{M}$ \\
\hline IAV-H1-1086F & CCC GTC YAT TCA ATC YAG AGG & $20 \mu \mathrm{M}$ \\
\hline IAV-H1-1180R & GGT GAT AAC CRT ACC ANC CAT C & $20 \mu \mathrm{M}$ \\
\hline IAV-H1-1190R & TCA TTT TGA TGR TGA TAA CCR TAC CA & $20 \mu \mathrm{M}$ \\
\hline IAV-H1-1155.1FAM & FAM-CAT YCC WGT CCA YCC YCC TTC AAT GAA-BHQ1 & $5 \mu \mathrm{M}$ \\
\hline \multicolumn{3}{|l|}{ H2 assays } \\
\hline IAV-H2-Mix 4-FAM & (this study) & \\
\hline IAV-H2-470-F & GAC ACA GCA YAC RAC AAC TGG & $15 \mu \mathrm{M}$ \\
\hline IAV-H2-601-R & GTG TTG TTG TAT GAT CYT TTR GCA A & $15 \mu \mathrm{M}$ \\
\hline IAV-H2-522-FAM & FAM-CCN TCA TTC TTC AGG AAC ATG GTY TGG-BHQ1 & $5 \mu \mathrm{M}$ \\
\hline IAV-H2-Mix 10-FAM & (this study) & \\
\hline IAV-H2-1075-F & CAA GRG GAT TGT TTG GRG CAA T & $15 \mu \mathrm{M}$ \\
\hline IAV-H2-1175-R & TGA TCC YTG RTC ATT GCT GTG & $15 \mu \mathrm{M}$ \\
\hline IAV-H2-1142-FAM & FAM-CCA RCC ATC AAC CAT YCC TTG CCA TCC-BHQ1 & $5 \mu \mathrm{M}$ \\
\hline \multicolumn{3}{|l|}{ H3 assays } \\
\hline IAV-H3-Mix 3-FAM & (this study) & \\
\hline IAV-H3-876-F & CTC CTC GGG GTT AYT TYA AAA T & $15 \mu \mathrm{M}$ \\
\hline IAV-H3-975-R & CCA TTT GGA GTG ATR CAT TCA GA & $15 \mu \mathrm{M}$ \\
\hline IAV-H3-934-FAM & FAM-TGC ATC TGA YCT CAT TAT TGA GCT TTT CCC-BHQ1 & $5 \mu \mathrm{M}$ \\
\hline IAV-H3-Mix 14-FAM & (this study) & \\
\hline IAV-H3-1667-F & TGG ATT TCC TTT GCC ATA TCA TG & $15 \mu \mathrm{M}$ \\
\hline IAV-H3-1784-R & ATR CAC TCA AAT GCA AAT GTT GCA & $15 \mu \mathrm{M}$ \\
\hline IAV-H3-1753-FAM & FAM-CTA ATG TTG CCT YTY TGG CAG GCC CAC AT-BHQ1 & $5 \mu \mathrm{M}$ \\
\hline \multicolumn{3}{|l|}{ H4 assay } \\
\hline IAV-H4-Mix 15-FAM & (this study) & \\
\hline IAV-H4-1586F & GAC TCA RGG ATA CAA RGA CAT & $15 \mu \mathrm{M}$ \\
\hline IAV-H4-1599F & AAG GAC ATC ATY CTY TGG ATT TC & $15 \mu \mathrm{M}$ \\
\hline IAV-H4-1686R & ACA AGC CCA CAA AAT RAA GGC & $15 \mu \mathrm{M}$ \\
\hline IAV-H4-1696R & TTC CRT TYT GAC AAG CCC ACA A & $15 \mu \mathrm{M}$ \\
\hline IAV-H4-1628FAM & FAM-TCC ATA TCA TGC TTY TTR CTC GTT GC-BHQ1 & $5 \mu \mathrm{M}$ \\
\hline \multicolumn{3}{|l|}{ H5 assays } \\
\hline IAV-H5-Mix 1-FAM & modified Spackman et al. ${ }^{25}$ CRL Weybridge & \\
\hline IAV-H5-1F & ACA TAT GAC TAC CCA CAR TAT TCA G & $25 \mu \mathrm{M}$ \\
\hline IAV-H5-1R & AGA CCA GCT AYC ATG ATT GC & $25 \mu \mathrm{M}$ \\
\hline IAV-H5-1FAM & Fam-TCW ACA GTG GCG AGT TCC CTA GCA-BHQ1 & $5 \mu \mathrm{M}$ \\
\hline IAV-H5a-Mix 1-FAM & (this study) & \\
\hline IAV-H5a-1658F & GTT CCC TAG YAC TGG CAA TCA T & $20 \mu \mathrm{M}$ \\
\hline IAV-H5a-1747R & AAT TCT ARA TGC AAA TTC TGC AYT G & $20 \mu \mathrm{M}$ \\
\hline IAV-H5a-1685FAM & FAM-CTG GTC TAT CYT THT GGA TGT GYT CCA ATG-BHQ1 & $5 \mu \mathrm{M}$ \\
\hline \multicolumn{3}{|l|}{ H6 assay } \\
\hline IAV-H6-Mix 8-FAM-MGB & (this study) & \\
\hline IAV-H6-1666F & CTT GGT GTG TAT CAA ATY CTT GC & $20 \mu \mathrm{M}$ \\
\hline IAV-H6-1776R & CAT TGA RCC ATT TGA RCA CAT CCA & $20 \mu \mathrm{M}$ \\
\hline
\end{tabular}




\begin{tabular}{|c|c|c|}
\hline Designation & Sequence $5^{\prime} \Rightarrow 3^{\prime}$ & $\begin{array}{l}\text { Concentration of primer and } \\
\text { probes in the primer-probe-mix }\end{array}$ \\
\hline IAV-H6-1693FAM-MGB & FAM-TAT AGT ACG GTA TCG AGC AGY CT-MGB & $5 \mu \mathrm{M}$ \\
\hline \multicolumn{3}{|l|}{ H7 assays } \\
\hline FLI-H7generic-2 & Kalthoff et al..$^{23}$ & \\
\hline IAV-HA7-1593-F & AYA GAA TAC AGA TWG ACC CAG T & $20 \mu \mathrm{M}$ \\
\hline IAV-HA7-1740-R & TAG TGC ACY GCA TGT TTC CA & $20 \mu \mathrm{M}$ \\
\hline IAV-HA7-1649-FAM & FAM-TGG TTT AGC TTC GGG GCA TCA TG -BHQ1 & $2.5 \mu \mathrm{M}$ \\
\hline IAV-H7-2.2-Mix-FAM & (this study) & \\
\hline IAV-HA7-1617-F & AAA TTG AGC AGY GGM TAC AAR GA & $25 \mu \mathrm{M}$ \\
\hline IAV-HA7-1707-R & AAA ACC ART CCC ATT RCA ATG GC & $25 \mu \mathrm{M}$ \\
\hline IAV-HA7-1649.1-FAM & FAM-TGG TTT AGC TTC GGG GCR TCA TGY TT_BHQ1 & $5 \mu \mathrm{M}$ \\
\hline FLI-H7-CODA & van Borm et al. ${ }^{26}$ & \\
\hline IAV-HA7-CODA-F & GYA GYG GYT ACA AAG ATG TG & $20 \mu \mathrm{M}$ \\
\hline IAV-HA7-CODA-R & GAA GAC AAG GCC CAT TGC AA & $20 \mu \mathrm{M}$ \\
\hline IAV-HA7-CODA-FAM & FAM-TGG TTT AGC TTC GGG GCA TCA TG-BHQ1 & $2.5 \mu \mathrm{M}$ \\
\hline \multicolumn{3}{|l|}{ H8 assay } \\
\hline IAV-H8-Mix 1-FAM & (this study) & \\
\hline IAV-H8-1604F & TAC AAA ATT CTY AGC ATY TAC AGT AC & $20 \mu \mathrm{M}$ \\
\hline IAV-H8-1677R & ATT ARA CCT CCA GCA AYC AGG A & $20 \mu \mathrm{M}$ \\
\hline IAV-H8-1654FAM & FAM-TGC CAA GCA RAG ACT GGC CGC CA-BHQ1 & $5 \mu \mathrm{M}$ \\
\hline \multicolumn{3}{|l|}{ H9 assay } \\
\hline IAV-H9-Mix 2-FAM & modified Monne et al. ${ }^{24}$ & \\
\hline IAV-H9-2F & ATG GGG TTT GCT GCC & $20 \mu \mathrm{M}$ \\
\hline IAV-H9-2R & TTA TAT ACA AAT GTT GCA YCTG & $20 \mu \mathrm{M}$ \\
\hline IAV-H9-2FAM & FAM-TTC TGG GCC ATG TCC AAT GG-BHQ1 & $5 \mu \mathrm{M}$ \\
\hline \multicolumn{3}{|l|}{ H10 assays } \\
\hline IAV-H10-Mix 5-FAM & (this study) & \\
\hline IAV-H10-991F & GTT GCT TGC WAC MGG AAT GAG & $15 \mu \mathrm{M}$ \\
\hline IAV-H10-1041F & GCC TGT TTG GDG CRA TAG C & $15 \mu \mathrm{M}$ \\
\hline IAV-H10-1122R & TTT TGR TGT CKG AAR CCA TAC CA & $15 \mu \mathrm{M}$ \\
\hline IAV-H10-1173R & ATA GCT GCY TGA GTA CTY TTG TA & $15 \mu \mathrm{M}$ \\
\hline IAV-H10-1092FAM & FAM-ACC ATY CCT TCC CAT CCR TTY TCT A-BHQ1 & $6.25 \mu \mathrm{M}$ \\
\hline IAV-H10-Mix 9-FAM & (this study) & \\
\hline IAV-H10-1567F & GAA ACT CTC TTC TGG VTA YAA AGA & $20 \mu \mathrm{M}$ \\
\hline IAV-H10-1695R & ATT GTG CAT CGC ATG TTT CCA T & $20 \mu \mathrm{M}$ \\
\hline IAV-H10-1601FAM & FAM-TGG TTT AGC TTC GGG GCR TCA TGY TT-BHQ1 & $5 \mu \mathrm{M}$ \\
\hline \multicolumn{3}{|l|}{ H11 assay } \\
\hline IAV-H11-Mix 1-FAM & (this study) & \\
\hline IAV-H11-1510F & ARG TYA GGA ATG GAA CAT ATG AYC A & $20 \mu \mathrm{M}$ \\
\hline IAV-H11-1723R & CAA ATG GTA CAT CTA CAT GAY CCA & $20 \mu \mathrm{M}$ \\
\hline IAV-H11-1626FAM & FAM-ATT TAC AGC TGC ATY GCA AGY AGT CT_BHQ1 & $12.5 \mu \mathrm{M}$ \\
\hline \multicolumn{3}{|l|}{ H12 assay } \\
\hline IAV-H12-Mix 3-FAM & (this study) & \\
\hline IAV-H12-1607F & AGC ATC TAC AGC AGT GTY GC & $20 \mu \mathrm{M}$ \\
\hline IAV-H12-1707R & CAG AAA GTA CAA CGA AYA TTT CCA & $20 \mu \mathrm{M}$ \\
\hline IAV-H12-1674FAM & FAM-CCG AAA ATG AAA CCC CCA ATA ATC ATG A-BHQ1 & $5 \mu \mathrm{M}$ \\
\hline \multicolumn{3}{|l|}{ H13 assay } \\
\hline IAV-H13-Mix 2-FAM & (this study) & \\
\hline IAV-H13-1241F & ATT GAC AAA ATG AAT GGR AAY TAT GAY TC & $20 \mu \mathrm{M}$ \\
\hline IAV-H13-1372R & AAG AAG YTT DGC ATT RTA TGA CCA & $20 \mu \mathrm{M}$ \\
\hline IAV-H13-1304FAM & $\begin{array}{l}\text { FAM-ATA AAY ATG CTY GCA GAY AGR ATA GAT GAY GC- } \\
\text { BHQ1 }\end{array}$ & $5 \mu \mathrm{M}$ \\
\hline \multicolumn{3}{|l|}{ H16 assay } \\
\hline IAV-H16-Mix 6-FAM-MGB & (this study) & \\
\hline IAV-H16-1589F & GGG ATA AAR TTG AAR ACT GAR GA & $20 \mu \mathrm{M}$ \\
\hline IAV-H16-1708R & ACT GCT RCA TGC CCA CAK TAT & $20 \mu \mathrm{M}$ \\
\hline IAV-H16-1635FAM-MGB & FAM-TTT AYA GYT GCA TTG CAA GCA G-MGB & $5 \mu \mathrm{M}$ \\
\hline \multicolumn{3}{|l|}{ Continued } \\
\hline
\end{tabular}




\begin{tabular}{|c|c|c|}
\hline Designation & Sequence $5^{\prime} \Rightarrow 3^{\prime}$ & $\begin{array}{l}\text { Concentration of primer and } \\
\text { probes in the primer-probe-mix }\end{array}$ \\
\hline \multicolumn{3}{|l|}{ N1 assay } \\
\hline IAV-N1-Mix 3-FAM & (this study) & \\
\hline IAV-N1-3-F & AGR CCT TGY TTC TGG GTT GA & $25 \mu \mathrm{M}$ \\
\hline IAV-N1-3-R & ACC GTC TGG CCA AGA CCA & $25 \mu \mathrm{M}$ \\
\hline IAV-N1-3-FAM & FAM-ATY TGG ACY AGT GGG AGC AGC AT-BHQ1 & $5 \mu \mathrm{M}$ \\
\hline \multicolumn{3}{|l|}{ N2 assay } \\
\hline IAV-N2-Mix 5.5 FAM-MGB & (this study) & \\
\hline IAV-N2-1367F & AGT CTG GTG GAC YTC AAA YAG & $30 \mu \mathrm{M}$ \\
\hline IAV-N2-1488R & AAT TGC GAA AGC TTA TAT AGV CAT & $30 \mu \mathrm{M}$ \\
\hline IAV-N2-1444.1FAM-MGB & FAM-CCA TCA GGC CAT GAG CCT-MGB & $5 \mu \mathrm{M}$ \\
\hline \multicolumn{3}{|l|}{ N3 assay } \\
\hline IAV-N3-Mix 2-FAM & (this study) & \\
\hline IAV-N3-1348F & AAY AGT ATA GTT ACT TTC TGY GG & $20 \mu \mathrm{M}$ \\
\hline IAV-N3-1422R & CCA ATG TTR GAA CCA TCH GG & $20 \mu \mathrm{M}$ \\
\hline IAV-N3-1373FAM & FAM-TAR ACA ATG AAC CTG GAT CGG GVA A-BHQ1 & $5 \mu \mathrm{M}$ \\
\hline IAV-N3-Mix 4-FAM & (this study) & \\
\hline IAV-N3-1348F & AAY AGT ATA GTT ACT TTC TGY GG & $20 \mu \mathrm{M}$ \\
\hline IAV-N3-1422R & CCA ATG TTR GAA CCA TCH GG & $20 \mu \mathrm{M}$ \\
\hline IAV-N3-1440R & TDT TAC TTG GGC ATD AAC CCA AT & $20 \mu \mathrm{M}$ \\
\hline IAV-N3-1373FAM & FAM-TAR ACA ATG AAC CTG GAT CGG GVA A-BHQ1 & $5 \mu \mathrm{M}$ \\
\hline \multicolumn{3}{|l|}{ N4 assay } \\
\hline IAV-N4-Mix 6-FAM & (this study) & \\
\hline IAV-N4-1335F & GAC YAG TGG TAG TAG YAT YGC & $20 \mu \mathrm{M}$ \\
\hline IAV-N4-1345F & AGT AGY ATT GCR TTY TGT GGT GTT & $20 \mu \mathrm{M}$ \\
\hline IAV-N4-1437R & AAA TYA CTT GTC TAT GTC AAA DGG & $20 \mu \mathrm{M}$ \\
\hline IAV-N4-1387FAM & FAM-TGG TCR TGG CCY GAT GGC GCT CT-BHQ1 & $5 \mu \mathrm{M}$ \\
\hline \multicolumn{3}{|l|}{ N5 assay } \\
\hline IAV-N5-Mix 5-FAM & (this study) & \\
\hline IAV-N5-1322F & AAG AGA GRA CWA GCA TTT GGA C & $15 \mu \mathrm{M}$ \\
\hline IAV-N5-1353F & CTC CAC KGT RTT TTG TGG TGT & $15 \mu \mathrm{M}$ \\
\hline IAV-N5-1421R & GGA AGA ATT GCK CCA TCA YC & $15 \mu \mathrm{M}$ \\
\hline IAV-N5-1426R & CAA AKG GAA GAA TTG CKC CAT CA & $15 \mu \mathrm{M}$ \\
\hline IAV-N5-1375FAM & FAM-TCM AGT GAG GTC CCA GGR TGG TC-BHQ1 & $5 \mu \mathrm{M}$ \\
\hline \multicolumn{3}{|l|}{ N6 assay } \\
\hline IAV-N6-Mix 3-FAM & (this study) & \\
\hline IAV-N6-10F & AGG GTG AAR ATG AAT CCA AAY CA & $20 \mu \mathrm{M}$ \\
\hline IAV-N6-14F & TGA ARA TGA ATC CAA ATC AGA AGA TAA & $20 \mu \mathrm{M}$ \\
\hline IAV-N6-97R & CAA TCC TAT YAG CAG RCT TAC TAC & $20 \mu \mathrm{M}$ \\
\hline IAV-N6-43FAM & FAM-TGC ATH TCA GCH ACA GGA ATG ACA CTA TC-BHQ1 & $5 \mu \mathrm{M}$ \\
\hline \multicolumn{3}{|l|}{ N7 assay } \\
\hline IAV-N7-Mix 1-FAM & (this study) & \\
\hline IAV-N7-1305F & GTT GAA TTA ATW AGA GGA AGR CC & $20 \mu \mathrm{M}$ \\
\hline IAV-N7-1430R & GAT YTG TGC CCC ATC RGG GA & $20 \mu \mathrm{M}$ \\
\hline IAV-N7-1383FAM & FAM-AGC CCA DTC YCA GTT GGG TCY GGT TC-BHQ1 & $5 \mu \mathrm{M}$ \\
\hline \multicolumn{3}{|l|}{ N8 assay } \\
\hline IAV-N8-Mix 1-FAM & (this study) & \\
\hline IAV-N8-1296F & TCC ATG YTT TTG GGT TGA RAT GAT & $20 \mu \mathrm{M}$ \\
\hline IAV-N8-1423R & GCT CCA TCR TGC CAY GAC CA & $20 \mu \mathrm{M}$ \\
\hline IAV-N8-1354FAM & FAM-TCH AGY AGC TCC ATT GTR ATG TGT GGA GT-BHQ1 & $10 \mu \mathrm{M}$ \\
\hline \multicolumn{3}{|l|}{ N9 assay } \\
\hline IAV-N9-Mix 11-FAM & Kalthoff et al. ${ }^{23}$ & \\
\hline IAV-N9-1363F & AGY ATA GTA TCR ATG TGT TCC AG & $20 \mu \mathrm{M}$ \\
\hline IAV-N9-1439R & AAG TAC TCT ATT TTA GCC CCA TC & $20 \mu \mathrm{M}$ \\
\hline IAV-N9-1393FAM & FAM-TTC CTB GGA CAA TGG AAC TGG CC-BHQ1 & $5 \mu \mathrm{M}$ \\
\hline \multicolumn{3}{|l|}{ Continued } \\
\hline
\end{tabular}




\begin{tabular}{|l|l|c|}
\hline Designation & Sequence $5^{\prime} \Rightarrow^{\prime} \mathbf{3}^{\prime}$ & $\begin{array}{c}\text { Concentration of primer and } \\
\text { probes in the primer-probe-mix }\end{array}$ \\
\hline IC assay & Hoffmann et al. ${ }^{16}$ & $5 \mu \mathrm{M}$ \\
\hline EGFP-Mix2- HEX & GAC CAC TAC CAG CAG AAC AC & $5 \mu \mathrm{M}$ \\
\hline EGFP-1-F & CTT GTA CAG CTC GTC CAT GC & $3.75 \mu \mathrm{M}$ \\
\hline EGFP-10-R & HEX-AGC ACC CAG TCC GCC CTG AGC A-BHQ1 & \\
\hline EGFP-HEX &
\end{tabular}

Table 3. Primers and probes used in this study.

A/mallard/Germany/R3036/2007 (H1N1), A/swine/Germany-NI/R369/2009 (H1N2) or A/swine/Germany/ AR1372/2015, whereas "H1 mix 27" failed to detect A/England/1/1951 (H1N1). Therefore, with a combination of both $\mathrm{H} 1$ mixes, all $\mathrm{H} 1$ isolates represented in the validation panel were correctly identified as representatives of the H1-subtype. While "H1 mix 3" proved to be exclusively specific for H1, "H1-mix 27" showed a weak cross-reactivity with $37 \mathrm{H} 6$ isolates and three isolates of the $\mathrm{H} 8$ subtype and therefore had a specificity of only $89.9 \%$. However, it should be noted that there was a difference in the Cq-values $\left(\Delta \mathrm{C}_{\mathrm{q}}\right)$ between the true specific and the false positives of more than 10 units when comparing the pan IAV-M1.2 assay to the false positive signals, and 9 units when comparing the specific assay to the false positive reacting assay results.

Both $\mathrm{H} 2$-detecting assays were specific for subtype $\mathrm{H} 2$ and identified 18 out of $18 \mathrm{H} 2$ isolates correctly, and an unspecific cross-reactivity was recorded for only one of the $\mathrm{H} 5$ isolates for the "IAV-H2-Mix 10 " ( $\Delta \mathrm{C}_{\mathrm{q}}>10$ generic assay, $\Delta \mathrm{C}_{\mathrm{q}}>8$ specific assays; Table 4). Furthermore, from a total of $40 \mathrm{H} 3$ isolates of the validation panel, 35 were detected by "IAV-H3- Mix 3", and 40 of 40 scored positive with the "IAV-H3-Mix 14" (Table 4). Additionally, all $17 \mathrm{H} 4$ isolates were correctly identified by "IAV-H4-Mix 15" (Table 4). Specific detection of all H5 sequences was achieved by "IAV-H5a-Mix 1" (88/88) and also approximated by the "IAV-H5-Mix 1" assay (87/88). Subtype H6 sequences (in a total of 61 isolates) were all correctly evaluated by the "IAV-H6-Mix 8 " assay (Table 4). Interestingly, for the complete identification of the H7 subtype sequences, three assays had to be implemented, providing the following coverage: "IAV-H7-CODA" detected 33 out of 35 correctly, with cross-reactivity with an $\mathrm{H} 5$ isolate, an $\mathrm{H} 10$ isolate and an $\mathrm{H} 15$ isolate; IAV-H7-Mix 2 detected 35 out of 35 correctly, with cross-reactions with six of the H10 isolates; "IAV-H7-Mix 2.2" detected all 35 isolates correctly. The nonspecific reactions again showed, in all cases, a $\Delta \mathrm{C}_{\mathrm{q}}>10$ compared to the specific reactions. Finally, the assays designed to detect the $\mathrm{H} 8, \mathrm{H} 9, \mathrm{H} 11, \mathrm{H} 12, \mathrm{H} 13$ and $\mathrm{H} 16$ subtypes reacted with the respective isolates and showed no cross-reactivity with any other subtypes. Nevertheless, both $\mathrm{H} 10$ assays exhibited nonspecific reactions in addition to the exact identification of all H10 subtypes: "IAV-H10-Mix 5" scored positive with 30 out of $40 \mathrm{H} 3$ isolates with Cq-values that were at least three units higher than the values of the "IAV-M1.2" assay and the specific assay; "IAV-H10-Mix 9" scored positive with 30 out of $35 \mathrm{H} 7$ isolates with a $\Delta$ Cq value of 5 compared to the IAV-M1.2 assay and $\Delta \mathrm{Cq}$ value of $>4$ compared to the specific assay. However, with the redundancy of the two tests, all $\mathrm{H} 10$ isolates could be correctly identified.

The reactivity of the NA-subtype specific assays showed a $100 \%$ match to the applied isolates, with no cross-reactivity (Table 5).

Diagnostic performance of RITA. The evaluation of diagnostic sensitivity was conducted using RNA from swab samples of wild birds or pigs. In general, subtyping was successful for those samples that exhibited Cq-values $<34$ with the pan IAV M1.2 assay. Out of 45 swab samples originating from wild aquatic birds, 45 samples exhibited (at least one) HA result and 44 samples (at least one) NA results by using RITA (Table 6). In 16 samples, additional reactivity with further subtypes was evident. Eleven of these additional reactivities apparently paralleled those already known from the validation panel; thus, no mixed IV infections were assumed for these samples. In contrast, mixed influenza infection was suspected in four samples because similar Cq-values for two or more HA and NA subtypes were obtained from multiple subtype assays. Verification of subtypes identified by RITA was achieved for the majority of bird samples by Sanger sequencing technology. However, samples showing Cq-values $>30$ in the generic IAV M1.2 assay did not regularly yield specific amplicons, especially for the HA, that were suitable for Sanger sequencing. The individual reaction patterns of all diagnostic samples are summarized in Table S2. Nasal swabs from swine were also used for subtype detection. For 15 out of 17 IAV positive samples, a subtype was unambiguously assignable by RITA (Table 6). One sample showed additional (cross-) reactivity, as previously seen from the validation panel. Specific subtypes identified by RITA were confirmed by subsequent Sanger sequencing (except for one sample for which no NA amplicon was obtained; Table S2). For one nasal swab sample, a mixed infection with more than one subtype was suspected (Table 6).

\section{Discussion}

The objective of this study was to develop and validate an RT-qPCR based subtyping tool for rapid and reliable direct subtyping of influenza A viruses from original sample material of avian and mammalian hosts. The finally validated 32-well RT-qPCR array, named RITA, allows the differentiation of 14 HA and 9 NA subtypes, as well as generic IAV RNA detection and an internal control system. The rare subtypes H14 and H15 were not implemented in this RITA version due to their minor relevance and to maintain an easy to manage format (e.g. using multiples of eight).

RT-qPCR-based nucleic acid detection techniques are routinely implemented throughout diagnostic laboratories, and the RITA protocol is easily established in such laboratories. The single tube duplex assay structure of RITA provides very high sensitivity for the individual assay, as well as a very good handling versatility, 


\begin{tabular}{|l|c|c|c|c|c|c|c|c|c|c|c|c|c|c|c|c|}
\hline RT-qPCR & H1 & H2 & H3 & H4 & H5 & H6 & H7 & H8 & H9 & H10 & H11 & H12 & H13 & H14 & H15 & H16 \\
\hline IAV-H1-Mix 3 & 51 & - & - & - & - & - & - & - & - & - & - & - & - & - & - & - \\
\hline IAV-H1-Mix 27 & 53 & - & - & - & - & $37^{*}$ & - & $3^{*}$ & - & - & - & - & - & - & - & - \\
\hline IAV-H2-Mix 4 & - & 18 & - & - & - & - & - & - & - & - & - & - & - & - & - & - \\
\hline IAV-H2-Mix 10 & - & 18 & - & - & $1^{*}$ & - & - & - & - & - & - & - & - & - & - & - \\
\hline IAV-H3-Mix 3 & - & - & 35 & - & - & - & - & - & - & - & - & - & - & - & - & - \\
\hline IAV-H3-Mix 14 & - & - & 40 & - & - & - & - & - & - & - & - & - & - & - & - & - \\
\hline IAV-H4-Mix 15 & - & - & - & 17 & - & - & - & - & - & - & - & - & - & - & - & - \\
\hline IAV-H5-Mix 1 & - & - & - & - & 87 & - & - & - & - & - & - & - & - & - & - & - \\
\hline IAV-H5a-Mix 1 & - & - & - & - & 88 & - & - & - & - & - & - & - & - & - & - & - \\
\hline IAV-H6-Mix 8 & - & - & - & - & - & 61 & - & - & - & - & - & - & - & - & - & - \\
\hline IAV-H7-CODA & - & - & - & - & $1 *$ & - & 33 & - & - & $1 *$ & - & - & - & - & $1 *$ & - \\
\hline IAV-H7-Mix 2 & - & - & - & - & - & - & 35 & - & - & $6 *$ & - & - & -- & - & - & - \\
\hline IAV-H7-Mix 2.2 & - & - & - & - & - & - & 35 & - & - & - & - & - & - & - & - & - \\
\hline IAV-H8-Mix 1 & - & - & - & - & - & - & - & 3 & - & - & - & - & - & - & - & - \\
\hline IAV-H9-Mix 2 & - & - & - & - & - & - & - & - & 31 & - & - & - & - & - & - & - \\
\hline IAV-H10-Mix 5 & - & - & $30^{\#}$ & - & - & - & - & - & - & 29 & - & - & - & - & - & - \\
\hline IAV-H10-Mix 9 & - & - & - & - & - & - & $30^{\dagger}$ & - & - & 29 & - & - & - & - & - & - \\
\hline IAV-H11-Mix 1 & - & - & - & - & - & - & - & - & - & - & 11 & - & - & - & - & - \\
\hline IAV-H12-Mix 3 & - & - & - & - & - & - & - & - & - & - & - & 1 & - & - & - & - \\
\hline IAV-H13-Mix 2 & - & - & - & - & - & - & - & - & - & - & - & - & 10 & - & - & - \\
\hline IAV-H16-Mix 6 & - & - & - & - & - & - & - & - & - & - & - & - & - & - & - & 4 \\
\hline total & 54 & 18 & 40 & 17 & 88 & 61 & 35 & 3 & 31 & 29 & 11 & 1 & 10 & 1 & 1 & 4 \\
\hline
\end{tabular}

Table 4. Reactivity of HA subtype-specific RT-qPCR assays used in RITA with the validation panel of 404 viral isolates. ${ }^{*} \Delta \mathrm{C}_{\mathrm{q}}$ between pan assay and false positive reactivity $\geq 10$ and $\Delta \mathrm{C}_{\mathrm{q}}$ between specific assay and false positive reactivity $\geq 8$. ${ }^{*} \Delta \mathrm{C}_{\mathrm{q}}$ between pan assay and false positive reactivity $\geq 3$ and $\Delta \mathrm{C}_{\mathrm{q}}$ between specific assay and false positive reactivity $\geq 3 .{ }^{\dagger} \Delta \mathrm{C}_{\mathrm{q}}$ between pan assay and false positive reactivity $\geq 5$; and $\Delta \mathrm{C}_{\mathrm{q}}$ between specific assay and false positive reactivity $\geq 4$.

\begin{tabular}{|l|c|c|c|c|c|c|c|c|c|}
\hline RT-qPCR & N1 & N2 & N3 & N4 & N5 & N6 & N7 & N8 & N9 \\
\hline IAV-N1-Mix 3 & 122 & - & - & - & - & - & - & - & - \\
\hline IAV-N2-Mix 5.5 & - & 118 & - & - & - & - & - & - & - \\
\hline IAV-N3-Mix 2 & - & - & 37 & - & - & - & - & - & - \\
\hline IAV-N3-Mix 4 & - & - & 37 & - & - & - & - & - & - \\
\hline IAV-N4-Mix 6 & - & - & - & 11 & - & - & - & - & - \\
\hline IAV-N5-Mix 5 & - & - & - & - & 6 & - & - & - & - \\
\hline IAV-N6-Mix 3 & - & - & - & - & - & 26 & - & - & - \\
\hline IAV-N7-Mix 1 & - & - & - & - & - & - & 37 & - & - \\
\hline IAV-N8-Mix 1 & - & - & - & - & - & - & - & 25 & - \\
\hline IAV-N9-Mix 11 & - & - & - & - & - & - & - & - & 16 \\
\hline total & 122 & 118 & 37 & 11 & 6 & 26 & 37 & 25 & 16 \\
\hline
\end{tabular}

Table 5. Reactivity of NA subtype-specific RT-qPCR assays used in RITA with the validation panel of 404 viral isolates.

because all 32 wells can be pipetted using a multiwell pipette and a single master mix per sample. This format also offers complete flexibility to modify existing or implement new assays targeting specific clades, HA cleavage sites (pathotyping for $\mathrm{H} 5$ and $\mathrm{H} 7$ subtypes; ${ }^{17}$, new geographical variants or assays for the identification of relevant pathogens of differential diagnostic importance. In the light of this flexibility, RITA can easily be adapted to the effects of evolutionary drift that may lead to IAV variants not only escaping vaccine-induced immunity but also routine diagnostics ${ }^{18}$. The current plate design resembling a low density PCR array format allows analysis of three samples in parallel per 96 well plate. The plates can be prepared in advance and stored at $-20^{\circ} \mathrm{C}$. A storage time of nine months did not lead to a loss of sensitivity. Using plates from the freezer, the overall setup and run time of RITA was less than three hours.

Recently, Elizalde and colleagues described a molecular subtyping approach for HA subtyping of IAV ${ }^{19}$, and the use of locked nucleic acids (LNA) within the probe sequence in this method rendered the RT-qPCR assays highly subtype-specific ${ }^{19}$. LNA probes are specific to such an extent that single nucleotide polymorphism (SNP) genotyping is possible ${ }^{20}$. However, with this implement, mismatches within the LNA probe binding region are less well tolerated, and the likelihood of false negative reactions increases. In addition, LNA-containing probes are more expensive than Taqman ${ }^{\circledR}$ or minor groove binding $\left(\mathrm{MGB}^{\circledR}\right)$ probes. Using SYBR green as a non-specific 


\begin{tabular}{|c|c|c|c|c|c|c|c|c|c|}
\hline \multirow[b]{3}{*}{ Sample origin } & \multirow{3}{*}{$\begin{array}{l}\text { total no. } \\
\text { tested }\end{array}$} & \multicolumn{5}{|c|}{ Subtyping by RITA } & & & \multirow{3}{*}{$\begin{array}{l}\text { Discrepancy RITA subtyping } \\
\text { and pan-HA/NA sequencing }\end{array}$} \\
\hline & & \multicolumn{2}{|c|}{$\begin{array}{l}\text { specific } \\
\text { results }\end{array}$} & \multicolumn{2}{|c|}{$\begin{array}{c}\text { presumed non-specific } \\
\text { reactivity }^{*}\end{array}$} & \multirow[t]{2}{*}{$\begin{array}{c}\text { mixed } \\
\text { infection }\end{array}$} & \multicolumn{2}{|c|}{$\begin{array}{l}\text { Subtyping by pan- } \\
\text { HA/NA sequencing }\end{array}$} & \\
\hline & & HA & NA & HA & NA & & HA & NA & \\
\hline wild bird swabs* & 45 & 45 & 44 & $16\left(11^{\dagger} / 5^{\ddagger}\right)$ & $1(0 / 1)$ & 4 & 32 & 40 & 0 \\
\hline swine nasal swabs & 17 & 17 & 17 & $1\left(1^{\dagger} / 0^{\ddagger}\right)$ & 0 & 1 & 17 & 16 & 0 \\
\hline total & 62 & 62 & 61 & $17(12 / 5)$ & $1(0 / 1)$ & 5 & 49 & 56 & 0 \\
\hline
\end{tabular}

Table 6. Diagnostic validation of RITA results from IAV-positive avian and porcine swab samples.

*oropharyngeal, cloacal or combined swab samples. ${ }^{*}$ RITA-nonspecific results are characterized by $\Delta$ Cq values according to details mentioned in table $4 .{ }^{\dagger}$ Nonspecific reactivity also detected with viruses of the validation panel. ${ }^{*}$ Nonspecific reactivity not previously seen in the validation panel. ${ }^{+}$In 13 and 6 samples, respectively, RITA could identify additional HA and NA subtypes (one or several) in comparison to the sequencing results. In one sample only the pan-NA sequencing procedure delivered a subtyping result. For more details see table S2.

detection tool, Tsukamoto and colleagues developed another RT-qPCR-based subtyping protocol for HA and NA of IAV ${ }^{21}$. This approach was successfully applied to identify NA subtypes from wild bird samples originating from a surveillance program ${ }^{22}$. However, SYBR green-based detection is less specific than probe-based detection technologies because the fluorescent substance binds to any double-stranded DNA molecule amplified during PCR.

With RITA, we attempted to find a perfect balance between highly sensitive broad reactivity and extreme subtype specificity with the use of Taqman ${ }^{\circledR} / \mathrm{MGB}$-based probes and, if necessary, multiple tests per subtype. Certain performance traits of single RT-qPCRs implemented in RITA were already demonstrated in citations ${ }^{15,16,23-26}$; in addition, some newly designed assays were compared with published protocols ${ }^{27}$, produced superior results, and, hence, were included in the RITA format. However, due to the wide-stretched spectrum of the virus panel used to validate RITA, comprising more than 400 isolates, comparative analysis of every newly designed assay against published Taqman assays was not feasible. The current version of RITA is certainly aimed at a best fit to Eurasian IAVs. Yet, the flexible basis of the array system allows the adaptation/inclusion/substitution of single RT-qPCRs to create RITA formats that serve specific needs e.g. according to geography (Eurasian versus American genotypes) or epidemiology (outbreak-adapted assays) or differential diagnostics.

RITA was validated using 404 influenza A virus isolates from both avian and mammalian hosts, and unequivocal subtype identification was completely successful on virus isolates. The need to design primers and probes that guaranteed the broadest within-subtype reactivity and the inherent prerequisite of RITA to apply the same cycling conditions to all 32 assays implemented produced few trade-off effects with respect to specificity for some of the assays. Slight tendencies for the co-amplification of non-targeted HA subtypes were observed for some of the selected RT-qPCRs. However, these false amplicons were usually detected with a much lower sensitivity and thus could easily be identified as false-positives when comparing the true positive subtype-specific reactions to the Cq-values obtained in the pan IAV M1.2 assay. Nevertheless, this propensity of the RITA assays reduces only the reliability to detect minor virus species (particularly H1, H10) in mixed IAV infections. The necessity of discerning co-amplification from mixed infection became apparent when examining clinical material from swine and avian species. The mixed infection with subtypes $\mathrm{H} 1$ and $\mathrm{N} 1$ and N2 detected in one porcine nasal swab demonstrated the extraordinary capacity of RITA to identify mixed infections in one sample. Sample materials originating from aquatic wild birds also gave evidence of mixed infections by diverse influenza A virus subtypes when examined by RITA (Table 6). Mixed infections are a precondition of genome reassortment ${ }^{28}$, but discerning mixed infections by standard Sanger sequencing technologies is seriously hampered ${ }^{29}$. The current RITA protocol in principal allows the identification of mixed IAV infections, although the interpretation of data may require certain expertise and experience to distinguish true co-infections with different IAV subtypes from unintended co-amplification or sample contamination. For instance, samples that exhibit similar Cq values for two or more HA and NA subtypes likely represent true co-infections. In contrast, if a single major subtype is identified and one or more very minor subtypes are present in the sample, no clear decision is possible unless other subtyping methods (virus isolation, next-generation sequencing) produce corroborating results. Therefore, and to keep in touch with the evolution and geographic dissemination of IAVs, the single RITA assays will have to be reassessed and improved on a regular basis.

Rapid and unequivocal IAV subtype identification, ideally even in clinical samples, is the prerequisite for monitoring the evolutionary dynamics and diversity of IAVs, with special emphasis on the One Health concept. With the use of RITA, the standard diagnostic cascade recommended for the detection and characterization of IAV in avian samples is reduced to a pretesting using a pan-influenza IAV-assay to verify presence of IAV RNA in the sample and a single RITA run. Future versions of RITA are intended to hold an "avian RITA", which includes cleavage site-specific assays for $\mathrm{H} 5 / \mathrm{H} 7$ pathotyping and the detection of avian pathogens of differential diagnostic relevance. An envisaged "mammalian Rita" can include assays for the differential diagnosis of influenza-like illnesses, while certain "avian" subtypes can be excluded from the array.

\section{References}

1. Vahlenkamp, T. W. \& Harder, T. C. Influenza virus infections in mammals. Berliner und Munchener tierarztliche Wochenschrift 119, 123-131 (2006).

2. Fields, B. N., Knipe, D. M. D. M.- \& Howley, P. M. Fields virology. 6th ed. edn, Vol. 1 (Wolters Kluwer, Lippincott Williams \& Wilkins, 2013).

3. Urbaniak, K. \& Markowska-Daniel, I. In vivo reassortment of influenza viruses. Acta Biochim Pol 61, 427-431 (2014). 
4. A revision of the system of nomenclature for influenza viruses: a WHO memorandum. Bulletin of the World Health Organization 58, 585-591 (1980).

5. Fouchier, R. A. et al. Characterization of a novel influenza A virus hemagglutinin subtype (H16) obtained from black-headed gulls. Journal of virology 79, 2814-2822, doi: 10.1128/JVI.79.5.2814-2822.2005 (2005).

6. Webster, R. G. \& Bean, W. J., Jr. Genetics of influenza virus. Annual review of genetics 12, 415-431, doi: 10.1146/annurev. ge.12.120178.002215 (1978).

7. Tong, S. et al. A distinct lineage of influenza A virus from bats. Proceedings of the National Academy of Sciences of the United States of America 109, 4269-4274, doi: 10.1073/pnas.1116200109 (2012).

8. Tong, S. et al. New world bats harbor diverse influenza A viruses. PLoS Pathog 9, e1003657, doi: 10.1371/journal.ppat.1003657 (2013).

9. Influenza (seasonal) Fact sheet $\mathrm{N}^{\circ} 211$, http://who.int/mediacentre/factsheets/fs211/en, (Date of access: 27/04/2016) (2014).

10. Girard, M. P., Cherian, T., Pervikov, Y. \& Kieny, M. P. A review of vaccine research and development: human acute respiratory infections. Vaccine 23, 5708-5724, doi: 10.1016/j.vaccine.2005.07.046 (2005).

11. OIE. Manual of Diagnostic Tests and Vaccines for Terrestrial Animals, http://www.oie.int/fileadmin/Home/fr/Health_standards/ tahm/2.03.04_AI.pdf, (Date of access: 27/04/2016) (2015).

12. Nagy, A. et al. MeltMan: Optimization, Evaluation, and Universal Application of a qPCR System Integrating the TaqMan qPCR and Melting Analysis into a Single Assay. PloS one 11, e0151204, doi: 10.1371/journal.pone.0151204 (2016).

13. Gall, A. et al. Rapid and highly sensitive neuraminidase subtyping of avian influenza viruses by use of a diagnostic DNA microarray. Journal of clinical microbiology 47, 2985-2988, doi: 10.1128/JCM.00850-09 (2009).

14. Gall, A. et al. Rapid haemagglutinin subtyping and pathotyping of avian influenza viruses by a DNA microarray. Journal of virological methods 160, 200-205, doi: 10.1016/j.jviromet.2009.05.004 (2009).

15. Hoffmann, B. et al. New real-time reverse transcriptase polymerase chain reactions facilitate detection and differentiation of novel A/H1N1 influenza virus in porcine and human samples. Berliner und Munchener tierarztliche Wochenschrift 123, 286-292 (2010).

16. Hoffmann, B., Depner, K., Schirrmeier, H. \& Beer, M. A universal heterologous internal control system for duplex real-time RT-PCR assays used in a detection system for pestiviruses. Journal of virological methods 136, 200-209, doi: 10.1016/j.jviromet.2006.05.020 (2006).

17. Hoffmann, B. et al. Rapid and highly sensitive pathotyping of avian influenza A H5N1 virus by using real-time reverse transcriptionPCR. Journal of clinical microbiology 45, 600-603, doi: 10.1128/JCM.01681-06 (2007).

18. Briand, F. X., Niqueux, E., Brochet, A. L., Hars, J. \& Jestin, V. Unusual H5N2 avian influenza virus escapes current detection. Journal of clinical microbiology 49, 2376-2377, doi: 10.1128/JCM.00479-11 (2011).

19. Elizalde, M. et al. Rapid molecular haemagglutinin subtyping of avian influenza isolates by specific real-time RT-PCR tests. Journal of virological methods 196, 71-81, doi: 10.1016/j.jviromet.2013.10.031 (2014).

20. Johnson, M. P., Haupt, L. M. \& Griffiths, L. R. Locked nucleic acid (LNA) single nucleotide polymorphism (SNP) genotype analysis and validation using real-time PCR. Nucleic Acids Res 32, e55, doi: 10.1093/nar/gnh046 (2004).

21. Tsukamoto, K. et al. SYBR green-based real-time reverse transcription-PCR for typing and subtyping of all hemagglutinin and neuraminidase genes of avian influenza viruses and comparison to standard serological subtyping tests. Journal of clinical microbiology 50, 37-45, doi: 10.1128/JCM.01195-11 (2012).

22. Latorre-Margalef, N. et al. Long-term variation in influenza A virus prevalence and subtype diversity in migratory mallards in northern Europe. Proc Biol Sci 281, 20140098, doi: 10.1098/rspb.2014.0098 (2014).

23. Kalthoff, D. et al. Nucleic acid-based detection of influenza A virus subtypes $\mathrm{H} 7$ and $\mathrm{N} 9$ with a special emphasis on the avian $\mathrm{H} 7 \mathrm{~N} 9$ virus. Euro surveillance : bulletin Europeen sur les maladies transmissibles = European communicable disease bulletin 19(10), $\mathrm{pii}=20731$, doi: $10.2807 / 1560-7917 . E S 2014.19 .10 .20731(2014)$.

24. Monne, I. et al. Development and validation of a one-step real-time PCR assay for simultaneous detection of subtype H5, H7, and H9 avian influenza viruses. Journal of clinical microbiology 46, 1769-1773, doi: 10.1128/JCM.02204-07 (2008).

25. Spackman, E. et al. Development of a real-time reverse transcriptase PCR assay for type A influenza virus and the avian H5 and H7 hemagglutinin subtypes. Journal of clinical microbiology 40, 3256-3260 (2002).

26. Van Borm, S. et al. Rapid detection of Eurasian and American H7 subtype influenza A viruses using a single TaqManMGB real-time RT-PCR. Avian diseases 54, 632-638 (2010).

27. Das, A. \& Suarez, D. L. Development and bench validation of real-time reverse transcription polymerase chain reaction protocols for rapid detection of the subtypes $\mathrm{H} 6, \mathrm{H} 9$, and $\mathrm{H} 11$ of avian influenza viruses in experimental samples. Journal of veterinary diagnostic investigation : official publication of the American Association of Veterinary Laboratory Diagnosticians, Inc 19, 625-634 (2007).

28. Dugan, V. G. et al. The evolutionary genetics and emergence of avian influenza viruses in wild birds. PLoS Pathog 4, e1000076, doi: 10.1371/journal.ppat.1000076 (2008).

29. Rejmanek, D., Hosseini, P. R., Mazet, J. A., Daszak, P. \& Goldstein, T. Evolutionary Dynamics and Global Diversity of Influenza A Virus. Journal of virology 89, 10993-11001, doi: 10.1128/JVI.01573-15 (2015)

\section{Acknowledgements}

We thank Patrick Zitzow, Aline Maksimov, and Diana Wessler at the Friedrich-Loeffler-Institut for their excellent technical assistance. Analysis of the swab samples from swine was supported by a grant to D. He. by IDT Biologika $\mathrm{GmbH}$. We would also like to thank Roche Diagnostics GmbH.

\section{Author Contributions}

B.H., M.B. and T.C.H. conceived and designed the study. B.H. and D.He. performed the experiments. B.H., T.C.H. D.Ho and M.B interpreted the data. D.Ho., T.C.H., B.H. and M.B. wrote the paper.

\section{Additional Information}

Supplementary information accompanies this paper at http://www.nature.com/srep

Competing financial interests: The authors declare no competing financial interests.

How to cite this article: Hoffmann, B. et al. Riems influenza a typing array (RITA): An RT-qPCR based low density array for subtyping avian and mammalian influenza a viruses. Sci. Rep. 6, 27211; doi: 10.1038/srep27211 (2016).

This work is licensed under a Creative Commons Attribution 4.0 International License. The images or other third party material in this article are included in the article's Creative Commons license, unless indicated otherwise in the credit line; if the material is not included under the Creative Commons license, users will need to obtain permission from the license holder to reproduce the material. To view a copy of this license, visit http://creativecommons.org/licenses/by/4.0/ 